\title{
Numerical uncertainty at mesoscale in a Lagrangian model in complex terrain
}

\author{
J. Brioude ${ }^{1,2}$, W. M. Angevine ${ }^{1,2}$, S. A. McKeen ${ }^{1,2}$, and E.-Y. Hsie ${ }^{1,2}$ \\ ${ }^{1}$ Cooperative Institute for Research in Environmental Sciences, University of Colorado at Boulder, Boulder, Colorado, USA \\ ${ }^{2}$ Chemical Sciences Division, Earth System Research Laboratory, NOAA, Boulder, Colorado, USA
}

Correspondence to: J. Brioude (jerome.brioude@noaa.gov)

Received: 13 April 2012 - Published in Geosci. Model Dev. Discuss.: 27 April 2012

Revised: 24 August 2012 - Accepted: 24 August 2012 - Published: 17 September 2012

\begin{abstract}
Recently, it has been shown that mass conservation in Lagrangian models is improved by using time-average winds out of Eulerian models. In this study, we evaluate the mass conservation and trajectory uncertainties in complex terrain at mesoscale using the FLEXPART Lagrangian particle dispersion model coupled with the WRF mesoscale model. The specific form of vertical wind used is found to have a large effect. Time average wind with time average sigma dot $(\dot{\sigma})$, instantaneous wind with geometric cartesian vertical wind $(w)$ and instantaneous wind with $\dot{\sigma}$ are used to simulate mixing ratios of a passive tracer in forward and backward runs using different time interval outputs and horizontal resolutions in California. Mass conservation in the FLEXPART model was not an issue when using timeaverage wind or instantaneous wind with $\dot{\sigma}$. However, mass was poorly conserved using instantaneous wind with $w$, with a typical variation of $25 \%$ within $24 \mathrm{~h}$.

Uncertainties in surface residence time (a backtrajectory product commonly used in source receptor studies or inverse modeling) calculated for each backtrajectory run were also analyzed. The smallest uncertainties were systematically found when using time-average wind. Uncertainties using instantaneous wind with $\dot{\sigma}$ were slightly larger, as long as the time interval of output was sufficiently small. The largest uncertainties were found when using instantaneous wind with $w$. Those uncertainties were found to be linearly correlated with the local average gradient of orography. Differences in uncertainty were much smaller when trajectories were calculated over flat terrain. For a typical run at mesoscale in complex terrain, $4 \mathrm{~km}$ horizontal resolution and $1 \mathrm{~h}$ time interval output, the average uncertainty and bias in surface residence time is, respectively, $8.4 \%$ and $-2.5 \%$ using time-average
\end{abstract}

wind, and $13 \%$ and $-3.7 \%$ using instantaneous wind with $\dot{\sigma}$ in complex terrain. The corresponding values for instantaneous wind with cartesian $w$ were $24 \%$ and $-11 \%$.

While the use of time-average wind systematically improves uncertainty in FLEXPART, the improvements are small, and therfore a systematic use of time-average wind in Lagrangian models is not necessarily required. Use of cartesian vertical wind in complex terrain, however, should be avoided.

\section{Introduction}

Lagrangian models have been used extensively to simulate atmospheric transport. Unlike Eulerian models, they do not suffer from numerical diffusion and can accurately calculate advection of fine scale structures (Stohl, 1998). At mesoscale, numerical diffusion in Eulerian models is not a major issue like in global models, and the benefit from using Lagrangian models comes from their flexibility and small computational cost compared to a full Eulerian model. Eulerian models are not always suitable for simulating inline transport of passive tracers (e.g., using forecast model reanalyses), while the same Lagrangian model can be used with different meteorological models to simulate the same experiment. Lagrangian models (like any off-line model) are therefore a powerful tool to generate representative ensembles of independent elements (e.g., Brioude et al., 2011). Furthermore, uncertainties in atmospheric transport due to uncertainties from modeled meteorology can also be assessed more accurately. 
However, Lagrangian models suffer from numerical uncertainties due to interpolation in space and time of simulated meteorological fields, and errors due to the conversion from the native vertical coordinate of the Eulerian model onto the vertical coordinate used in the Lagrangian model. Furthermore, mass conservation could be an issue when the vertical velocity is not mass balanced with horizontal winds.

Recently, Nehrkorn et al. (2010) analyzed the mass conservation in a Lagrangian model using instantaneous or timeaverage wind from a mesoscale Eulerian model. They used mass weighted time average wind (horizontal and vertical) and instantaneous wind for driving the STILT Lagrangian model using the Weather Research and Forecast (WRF) Eulerian forecast model at a horizontal resolution of $12 \times 12 \mathrm{~km}^{2}$ and a time resolution of $1 \mathrm{~h}$. They found that the mass conservation using instantaneous wind from WRF was very poor, and using time average wind was "crucial in improving the mass conservation properties of the coupled modeling system". The authors suggested that those large differences in mass conservation were due to the fact that mass weighted time average winds were less subject to errors in interpolations and vertical coordinate transformations than instantaneous winds.

Grell et al. (2004) have shown that wind variability can be captured by instantaneous winds, as long as the output time interval is short enough for a given horizontal resolution. For instance, a 3-km horizontal resolution run would need an output interval of $15 \mathrm{~min}$ to capture $50 \%$ of the wind variability in the case of a cold front passage. Therefore, it is important to understand how trajectory uncertainties and mass conservation vary with horizontal resolution and output interval when using instantaneous wind or time-average wind. Theoretically, the mass conservation should be identical when using time-average wind or instantaneous wind when a very short output interval is used, the lower limit being the time step within the Eulerian model. Furthermore, vertical coordinate transformation between the native Eulerian model coordinate and the Lagrangian model coordinate can be responsible for additional uncertainties. One can expect larger uncertainties in Lagrangian trajectories in complex terrain where errors in vertical coordinate transformation are the largest.

In this article, we present an analysis of mass conservation and trajectory uncertainties using time-average and instantaneous winds for different output time intervals, vertical velocity variables, and horizontal grid spacing. The Lagrangian trajectories were calculated by a modified version of the FLEXPART Lagrangian particle dispersion model with WRF output using (1) the instantaneous wind with cartesian vertical velocity, (2) the instantaneous wind with sigma dotas vertical velocity, and (3) mass weighted time averaged horizontal and vertical winds. Our goal is to evaluate the impact of those different types of wind on the uncertainties of trajectory calculations that could reduce the accuracy of typical applications of Lagrangian particle dispersion model runs, such as source receptor relationships for inverse modeling or mixing ratio estimates of a tracer. We do not evaluate the accuracy of the wind and meteorological fields from the Eulerian model itself in this paper.

Section 2 presents in detail the WRF and FLEXPART model configurations. Section 3 presents results on wind divergence, mass conservation and uncertainties. A discussion and conclusion is given in Sect. 4.

\section{Models}

\subsection{WRF}

We used a configuration of the Weather Research and Forecasting (WRF) mesoscale research model version 3.3 that uses mass weighted variables (ARW core) with nested grids at 36, 12 and $4 \mathrm{~km}$ horizontal spacing with 60 vertical levels. The model was initialized at 12:00 UTC and run for $36 \mathrm{~h}$ each day. The model was initialized with ERA-interim reanalysis (Simmons et al., 2007). The MYJ planetary boundary layer (PBL) scheme and Noah land surface scheme were used. Other significant physics options were Eta-Ferrier microphysics, RRTM-G (Rapid Radiative Transfer Model) longwave and Dudhia shortwave radiation. and Grell-Devenyi cumulus (outer domain only).

Angevine et al. (2012) present detailed evaluations of this simulation (their run EM4N). It should be noted that the fidelity of the simulated winds to reality does not influence the results presented here.

The WRF output is on a Arakawa C-grid with terrain following pressure based sigma levels (called eta levels in Skamarock et al., 2008). It includes instantaneous horizontal wind on sigma levels (variable names $U$ and $V$ in $\mathrm{ms}^{-1}$ ), a geometric cartesian vertical velocity $\left(W\right.$ in $\mathrm{ms}^{-1}$ ) that we call $w$, an instantaneous sigma dot vertical velocity (WW in $\mathrm{s}^{-1}$ ) that we call $\dot{\sigma}$, and mass weighted time-average winds on sigma levels (AVGFLX_RUM, AVGFLX_RVM, AVGFLX_WWM). The time-average vertical wind is a mass weighted time average sigma dot, and therefore its vertical coordinate transformation in FLEXPART is equivalent to the one applied on $\dot{\sigma}$.

The $4 \mathrm{~km}$ and $12 \mathrm{~km}$ grid spacing domains, and $30 \mathrm{~min}, 1 \mathrm{~h}$ and $2 \mathrm{~h}$ time interval outputs are used to run FLEXPART.

\subsection{FLEXPART}

We used a version of the FLEXPART Lagrangian particle dispersion model modified to use WRF output (Fast and Easter, 2006; Brioude et al., 2011). The model uses the horizontal grid coordinates from WRF, but uses a terrain following cartesian coordinate for vertical levels, and therefore a vertical interpolation for the different meteorological fields has to be performed. To run the model with an accurate representation of the turbulent mixing in the PBL, the time step in FLEXPART is calculated dynamically depending on the 
values of a Lagrangian timescale, vertical velocity and mixing height (see Eq. 19 in Stohl et al., 2005). An upper limit was fixed at $180 \mathrm{~s}$ for horizontal mixing and $36 \mathrm{~s}$ for vertical mixing. On average, the time scale was $100 \mathrm{~s}$ for horizontal mixing and $20 \mathrm{~s}$ for vertical mixing. The horizontal resolution of the FLEXPART output domain was $8 \times 8 \mathrm{~km}^{2}$.

The Hanna turbulent scheme (Hanna, 1982) was used to represent turbulent mixing in the boundary layer ${ }^{1}$. Parameters used in the Hanna scheme are calculated internally by FLEXPART (e.g., PBL mixing height, friction velocity, etc.) rather than retrieved directly from the WRF model output to improve consistency with the wind fields. See Stohl et al. (2005) for further details.

We chose California for our analysis. Gradients in orography in this region can be particularly strong. The CALNEX campaign took place in California in 2010, with several flights dedicated to characterizing surface emissions in Central Valley and the Los Angeles basin. California is a difficult region to simulate due to complex terrain, differences in surface moisture between Central Valley and Los Angeles Basin, sea breeze, etc. See Angevine et al. (2012) for further details.

To examine mass consistency, we compared forward and backward trajectories. Forward trajectories were simulated over $24 \mathrm{~h}$. Each trajectory carried an equal mass of a passive tracer based on an arbitrary homogeneous surface flux. Overall, 15 million trajectories were released homogeneously over the surface domain, and continuously from May 16th to May 20th 2010. Mixing ratios of this tracer were output every hour within grid cells with a horizontal resolution of $8 \times 8 \mathrm{~km}^{2}$ and within 14 vertical layers stretched between the surface and $4 \mathrm{~km}$ in altitude.

Backward trajectories were calculated 4 times a day between 17 and 19 May over $24 \mathrm{~h}$ from 108 different geographical locations (every 5 grid cells in $\mathrm{x}$ direction, and every 10 grid cells in y direction) and at 3 different vertical levels (between 0 and $100 \mathrm{~m}$, between 200 and $400 \mathrm{~m}$ and between 800 and $1100 \mathrm{~m}$ ) that match grid cell positions in the forward run output domain. 5000 particles are released for each time and location. For each set of trajectories, the output consists of a residence time in the surface layer weighted by the atmospheric density (the so-called footprint). When this output is combined with a surface flux, one can calculate a mixing ratio for each set of backtrajectories (e.g., Brioude et al., 2011). We used the same surface flux of the homogeneous tracer used in the forward run to calculate a mixing ratio at each starting location of the backtrajectories.

Lin et al. (2003) have shown that asymmetry between backward and forward runs arise mostly from errors in the mass balance of the driving winds. In our case, perfectly

\footnotetext{
${ }^{1}$ To date, an option that uses 3-D fields of Turbulent Kinetic Energy is also available in the FLEXPART-WRF version. However, the well mixed criterion is not satisfied using this option, and is therefore not recommended at the time of submission.
}

mass balanced winds would give the same mixing ratio at each location from either forward or backward experiments, assuming that the Hanna turbulent scheme satisfy the well mixed criterion. Overall, 3888 mixing ratios are calculated for each experiment. In Sect. 3, we present differences using different wind treatments, different grid spacing $(4 \mathrm{~km}$ and $12 \mathrm{~km}$ ) and different time interval outputs $(30 \mathrm{~min}, 1 \mathrm{~h}$ and $2 \mathrm{~h})$.

\section{Mass conservation and uncertainties}

\subsection{Wind divergence}

According to Skamarock et al. (2008), the mass in the WRF model is fully conserved relative to the sixth-order RungeKutta advection algorithm. Therefore, horizontal and vertical winds in the WRF outputs are considered mass conservative. Loss of mass conservation in a Lagrangian model should arise from unit conversion, vertical coordinate transformation and uncertainties in the turbulent scheme used. Mass conservation in FLEXPART can be calculated for each grid cell using the mass continuity equation based on the wind divergence in cartesian coordinate.

$\frac{1}{\rho} \frac{\partial \rho}{\partial t}=\frac{1}{\rho}\left(\frac{\partial \rho u}{\partial x}+\frac{\partial \rho v}{\partial y}+\frac{\partial \rho W}{\partial z}\right)$.

With $u, v$ and $W$ being the horizontal and vertical wind in cartesian terrain following coordinate in FLEXPART, $\rho$ being the air density. We calculated it after converting the WRF wind on sigma coordinates onto the terrain following cartesian vertical coordinate within FLEXPART. The gradients are calculated using a central finite difference method.

Figure 1 presents the evaluation of Eq. (1) for each grid cell at $50 \mathrm{~m}$ above ground on 16 May at $00 z$ using mass weighted time average winds, instantaneous winds with cartesian geometric vertical velocity $(w)$ and instantaneous winds with sigma dot $(\dot{\sigma})$. The differences in divergence at $50 \mathrm{~m}$ are representative of the differences found within the PBL for other dates. The wind divergence is small and almost identical when using time-average wind or instantaneous winds with $\dot{\sigma}$. Non-zero values come from uncertainties in converting the winds onto the FLEXPART vertical coordinate, or due to non-hydrostatic terms in the WRF model. The wind divergence is larger and more variable using the instantaneous wind with $w$, especially in complex terrain. However, no differences are found between wind divergences calculated by the three methods over flat terrain (either over the ocean or Central Valley). The strong divergence in complex terrain is the consequence of uncertainties in $w$ related to variations in orography.

To convert $w$ from a geometric vertical coordinate onto a terrain following coordinate (either on cartesian or sigma coordinate), a corrective factor has to be applied. This 

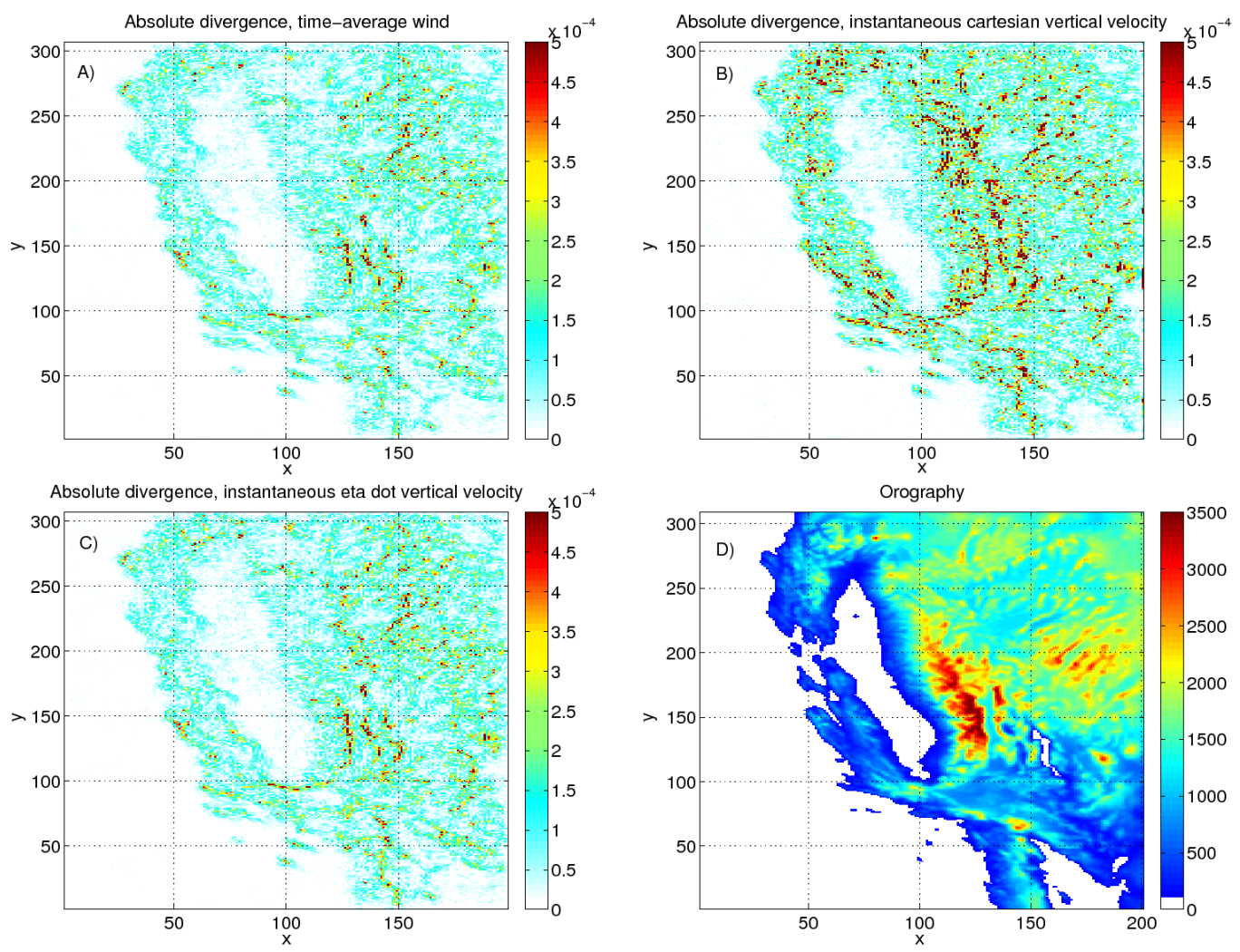

Fig. 1. Absolute wind divergence at about $50 \mathrm{~m}$ in altitude using (a) time-average wind, (b) instantaneous wind with cartesian vertical velocity, and (c) instantaneous wind with sigma dot (in $\mathrm{s}^{-1}$ ). (d) Orography (in $\mathrm{m}$ ).

corrective factor is of the form:

$W=w-u \frac{\partial Z}{\partial x}-v \frac{\partial Z}{\partial y}$,

with $Z$ being the orography from WRF output, and $W$ being the terrain following vertical velocity.

There are two possible reasons for the large differences in wind divergence using $w$ and $\dot{\sigma}$. The first possibility is that $w$ cannot be accurately converted into a vertical velocity on a terrain following cartesian coordinate within a Lagrangian model. For instance, the terms $u \mathrm{~d} Z / \mathrm{d} x$ and $v \mathrm{~d} Z / \mathrm{d} y$ are underestimated within FLEXPART as they need to be calculated over $2 \mathrm{~d} x$ and $2 \mathrm{~d} y$. Within WRF, the horizontal grid is staggered. Uncertainties from coordinate transformation is much smaller when using $\dot{\sigma}$ (or the time-average equivalent) because it involves a conversion from a terrain following pressure based sigma coordinate (in WRF) onto a terrain following cartesian coordinate (in FLEXPART). In this case, a corrective factor involves only horizontal differences in geopotential between those 2 terrain following coordinates, and is very small in magnitude. This fact is valid for any offline Lagrangian model with a terrain following coordinate. This deficiency does not occur when using fully Lagrangian models.
A second reason might come from the fact that in the WRF model, the mass weighted $w$ is a prognostic variable, while the mass weighted $\dot{\sigma}$ is a diagnostic variable used in the mass continuity equation resolved by the model that involves mass weighted $u$ and $v$. Since $w$ is a prognostic variable, it might have additional uncertainties due to uncertainties in the prognostic equation in steep terrain that $\dot{\sigma}$ might not have. It is also important to notice that the differences in wind divergence found with this particular configuration might be different using other PBL schemes or using a stronger damping coefficient for acoustic waves. However, we are confident that using $\dot{\sigma}$ would systematically improve the wind divergence for any configuration.

Nehrkorn et al. (2010) compared mass weighted time average wind on sigma levels with instantaneous wind using geometric cartesian $w$ from the WRF output. Their analysis involves the same errors in vertical coordinate transformations that have been explained above, which could have contributed to the differences they found on mass conservation.

As vertical wind is not necessarily present in Eulerian model outputs or with uncertainties that cannot be accurately corrected within Lagrangian models, we added the possibility to use a vertical velocity calculated internally in 

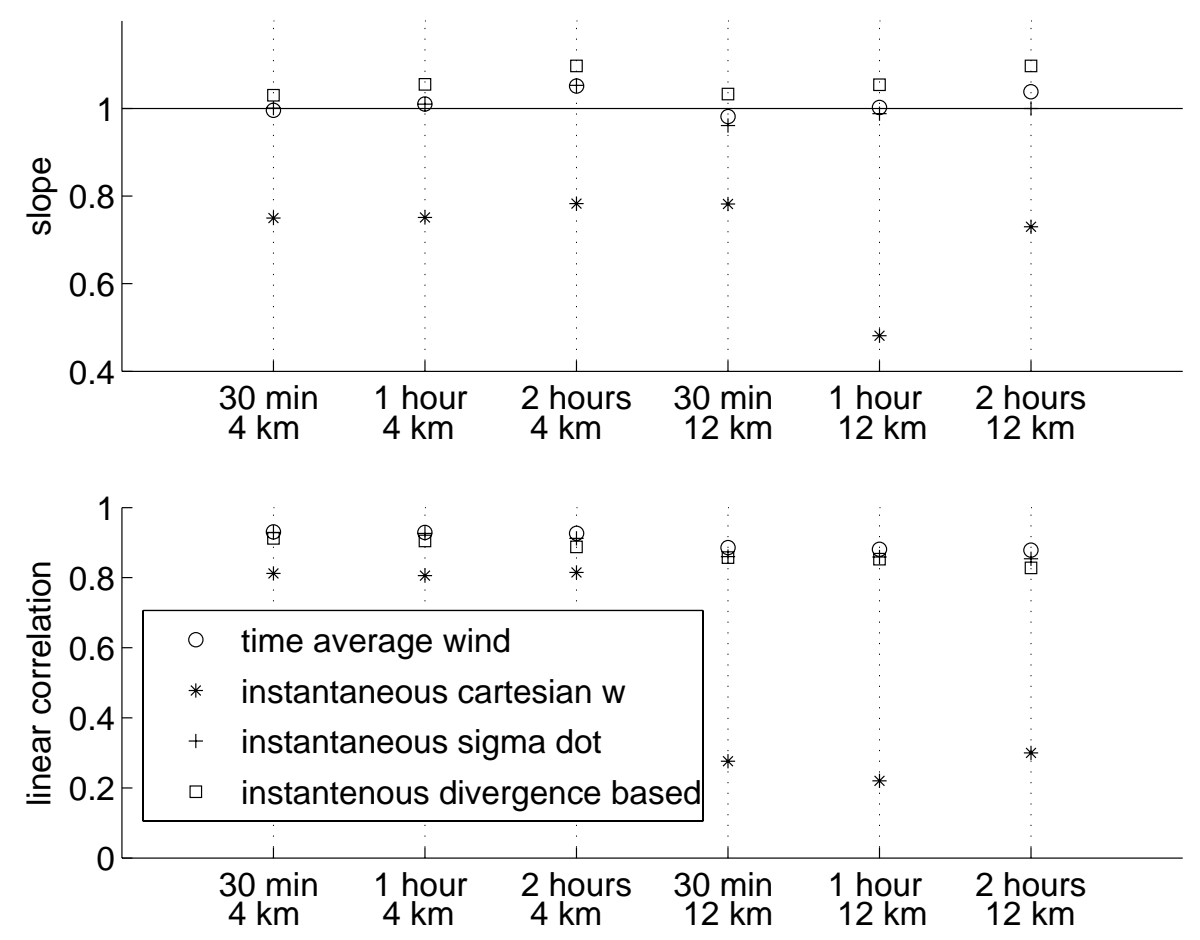

Fig. 2. (Top) slope and (bottom) linear correlation of mixing ratio between forward and backward runs, for 6 different experiments using 4 types of winds. See Table 1 and Sect. 3.2 for details.

FLEXPART based on Eq. (1) assuming hydrostatic conditions, that we call $W_{f}$.

In the following subsections, we show results of mass conservation and uncertainty using time-average wind, and instantaneous wind using $w, \dot{\sigma}$ and $W_{f}$.

\subsection{Mass conservation}

In this section, we present comparisons of mixing ratio of a passive tracer at different locations based on forward runs and backward runs calculated over $24 \mathrm{~h}$. A perfectly mass conserving model should be reversible in time, in a sense that backward and forward trajectories should give the same results (Lin et al., 2003). A mass conserving model should therefore have identical mixing ratios based on forward and backward runs. A distribution of mixing ratios from forward and backward runs should have a slope of 1 , and a linear correlation of 1 . These tests involving mixing ratios are equivalent to the count of trajectories between backward and forward runs in Nehrkorn et al. (2010).

It is important to notice that a source of uncertainty beside transport and mixing in FLEXPART is the limited number of trajectories used in this study. We used 5000 trajectories per location in the backward runs, and 15 millions of trajectory in each forward runs. Uncertainties between backward and forward runs at each location could come from the limited number of trajectories to calculate mixing ratios within each grid cells. However, it should only affect the linear cor- relations between mixing ratios from backward and forward runs, and should not modify the slopes.

Figure 2 and Table 1 present the slopes and linear correlations calculated in complex terrain using the 4 different types of wind, and using $30 \mathrm{~min}, 1 \mathrm{~h}$, or $2 \mathrm{~h}$ output interval and $4 \mathrm{~km}$ or $12 \mathrm{~km}$ horizontal grid. A slope lower than 1 means a loss of mass in backward runs or gain of mass in forward runs.

Using $30 \mathrm{~min}$ time interval output with $4 \mathrm{~km}$ resolution, the slopes from the runs that use mass weighted time average wind and instantaneous wind with $\dot{\sigma}$ are very close to 1 (0.996 and 1.001, respectively). It means that FLEXPART loses (or gains) less than $0.4 \%$ and $0.1 \%$ of mass within $24 \mathrm{~h}$. Their linear correlations are the largest found $(0.931$ and 0.929) among the different experiments. On average, the slopes from the runs using time-average wind and instantaneous wind with $\dot{\sigma}$ are different by less than $2 \%$, except for the experiment using $12 \mathrm{~km}$ resolution and $2 \mathrm{~h}$ output $(4 \%)$. These results show that differences in mass conservation in FLEXPART between runs using mass weighted time average wind and instantaneous wind with $\dot{\sigma}$ is insignificant.

Using instantaneous wind with the cartesian vertical velocity $w$, the best slope found is 0.75 using $4 \mathrm{~km}$ resolution and 30 min output, with a linear correlation of 0.81 . The slope ranges from $0.48-0.78$ among the other experiments. It means that the mass within FLEXPART varies by about $25 \%$ within $24 \mathrm{~h}$ using instantaneous wind with $w$. Furthermore, 
Table 1. Mass conservation experiments (in complex terrain) using time-average ("mean”) wind, instantaneous wind with cartesian wind $w$ from WRF ("cartesian"), instantaneous wind with sigma dot from WRF, and instantaneous wind with vertical wind calculated internally in FLEXPART, for 3 different output intervals and 2 WRF horizontal grid spacings.

\begin{tabular}{lcccc}
\hline & \multicolumn{2}{c}{$4 \mathrm{~km}$} & \multicolumn{2}{c}{$12 \mathrm{~km}$} \\
Experiment & Geometric slope & Linear correlation & Geometric slope & Linear correlation \\
\hline Mean 0.5 h & 0.996 & 0.931 & 0.982 & 0.886 \\
Cartesian 0.5 h & 0.750 & 0.812 & 0.782 & 0.276 \\
sigma dot 0.5 h & 1.001 & 0.929 & 0.961 & 0.86 \\
Internal 0.5 h & 1.03 & 0.912 & 1.033 & 0.857 \\
Mean 1 h & 1.01 & 0.929 & 1.002 & 0.881 \\
Cartesian 1 h & 0.751 & 0.806 & 0.481 & 0.220 \\
sigma dot 1 h & 1.01 & 0.926 & 0.988 & 0.859 \\
Internal 1 h & 1.055 & 0.905 & 1.054 & 0.853 \\
Mean 2 h & 1.051 & 0.926 & 1.038 & 0.878 \\
Cartesian 2 h & 0.783 & 0.815 & 0.73 & 0.30 \\
sigma dot 2 h & 1.053 & 0.912 & 1.000 & 0.854 \\
Internal 2 h & 1.097 & 0.888 & 1.097 & 0.828 \\
\hline
\end{tabular}

mixing ratios are poorly correlated using $12 \mathrm{~km}$ resolution (correlation less than 0.3 ).

Runs using instantaneous wind with $W_{f}$ are significantly less mass conserving than using mass weighted time average wind or instantaneous wind with $\dot{\sigma}$, but much better than using cartesian vertical velocity $w$. For a typical time interval of $1 \mathrm{~h}$, the slope is about 1.05 , which means that the mass varies by $5 \%$ within $24 \mathrm{~h}$ in the model.

The results found using instantaneous wind with $w$ confirm the results found in Nehrkorn et al. (2010). In complex terrain, the mass variation is about $25 \%$ within $24 \mathrm{~h}$, and therefore, the use of $w$ should be avoided. However, we hardly see a benefit using time-average wind instead of instantaneous wind (using $\dot{\sigma}$ ) in terms of mass conservation, and therefore disagree with the conclusions given by Nehrkorn et al. (2010) that time-average wind is crucial to have mass conservation within a Lagrangian model at mesoscale. Those results are in agrement with the conclusions found in Sect. 3.1.

\subsection{Surface residence time uncertainties}

In this section, trajectory uncertainties in each experiment are investigated by comparing the surface residence time found in each grid cell for each backward run. Those biases and uncertainties can be used to estimate, for instance, uncertainties involved in source-receptor relationships.

Figure 3 and Table 2 present relative standard deviation (\%) and bias (\%) for each experiment relatively to the best FLEXPART backward run, namely the run using mass weighted time average winds at $4 \mathrm{~km}$ resolution and $30 \mathrm{~min}$ output interval. The relative standard deviation is an estimate of the average uncertainty of residence time within each grid cell, and the relative bias is an estimate of the average difference of surface residence time between each run and the reference run. The relative biases are the consequence of dif-
Table 2. Average relative error of the surface residence time in each grid cell relatively to the FLEXPART runs that use time-average wind at $4 \mathrm{~km}$ and $0.5 \mathrm{~h}$ time interval output.

\begin{tabular}{lcccc}
\hline & \multicolumn{2}{c}{$4 \mathrm{~km}$} & \multicolumn{2}{c}{$12 \mathrm{~km}$} \\
& Relative std (\%) & Bias (\%) & Relative std (\%) & Bias (\%) \\
\hline Mean 0.5 h & N/A & N/A & $28 \%$ & $-4.6 \%$ \\
Cartesian 0.5 h & $19 \%$ & $-8.6 \%$ & $40 \%$ & $+9.2 \%$ \\
sigma dot 0.5 h & $6.5 \%$ & $-1.5 \%$ & $32 \%$ & $-9.6 \%$ \\
Internal 0.5 h & $10 \%$ & $-1.0 \%$ & $32 \%$ & $-7.1 \%$ \\
Mean 1 h & $8.4 \%$ & $-2.5 \%$ & $31 \%$ & $-7.3 \%$ \\
Cartesian 1h & $24 \%$ & $-11 \%$ & $43 \%$ & $+6.4 \%$ \\
sigma dot 1h & $13 \%$ & $-3.7 \%$ & $34 \%$ & $-12 \%$ \\
Internal 1h & $16 \%$ & $-3.6 \%$ & $35 \%$ & $-11 \%$ \\
Mean 2h & $23 \%$ & $-5.1 \%$ & $37 \%$ & $-11 \%$ \\
Cartesian 2h & $37 \%$ & $-16 \%$ & $53 \%$ & $+1.1 \%$ \\
sigma dot 2h & $29 \%$ & $-9.1 \%$ & $43 \%$ & $-18 \%$ \\
Internal 2h & $31 \%$ & $-9.3 \%$ & $46 \%$ & $-18 \%$ \\
\hline
\end{tabular}

ferences in vertical transport and differences in mass conservation between each run and the reference run. Errors in vertical velocity impact mostly the bias, while errors in the horizontal wind impact the relative standard deviation.

Time average wind has systematically the best uncertainty and bias. For a typical time interval output of $1 \mathrm{~h}$, the uncertainty is $8.4 \%$ at $4 \mathrm{~km}$ and $31 \%$ at $12 \mathrm{~km}$ spacing, while the bias is $-2.5 \%$ at $4 \mathrm{~km}$ resolution and $-7.3 \%$ at $12 \mathrm{~km}$ spacing compared to the reference run. Uncertainties and biases using the instantaneous wind with $\dot{\sigma}$ are the second lowest. For a typical time interval output of $1 \mathrm{~h}$ at $4 \mathrm{~km}$ resolution, the uncertainty is $13 \%$ and bias is $-3.7 \%$. They are larger by $4.6 \%$ and $1.2 \%$, respectively, compared to the time average wind run. At $12 \mathrm{~km}$ resolution, the uncertainty is $34 \%$ and bias is $-12 \%$ which is larger by $3 \%$ and $5.3 \%$, respectively than the values found using time average wind. On average, the difference in relative bias between time average wind and instantaneous wind using $\dot{\sigma}$ vary from -1.2 to $-7 \%$. 

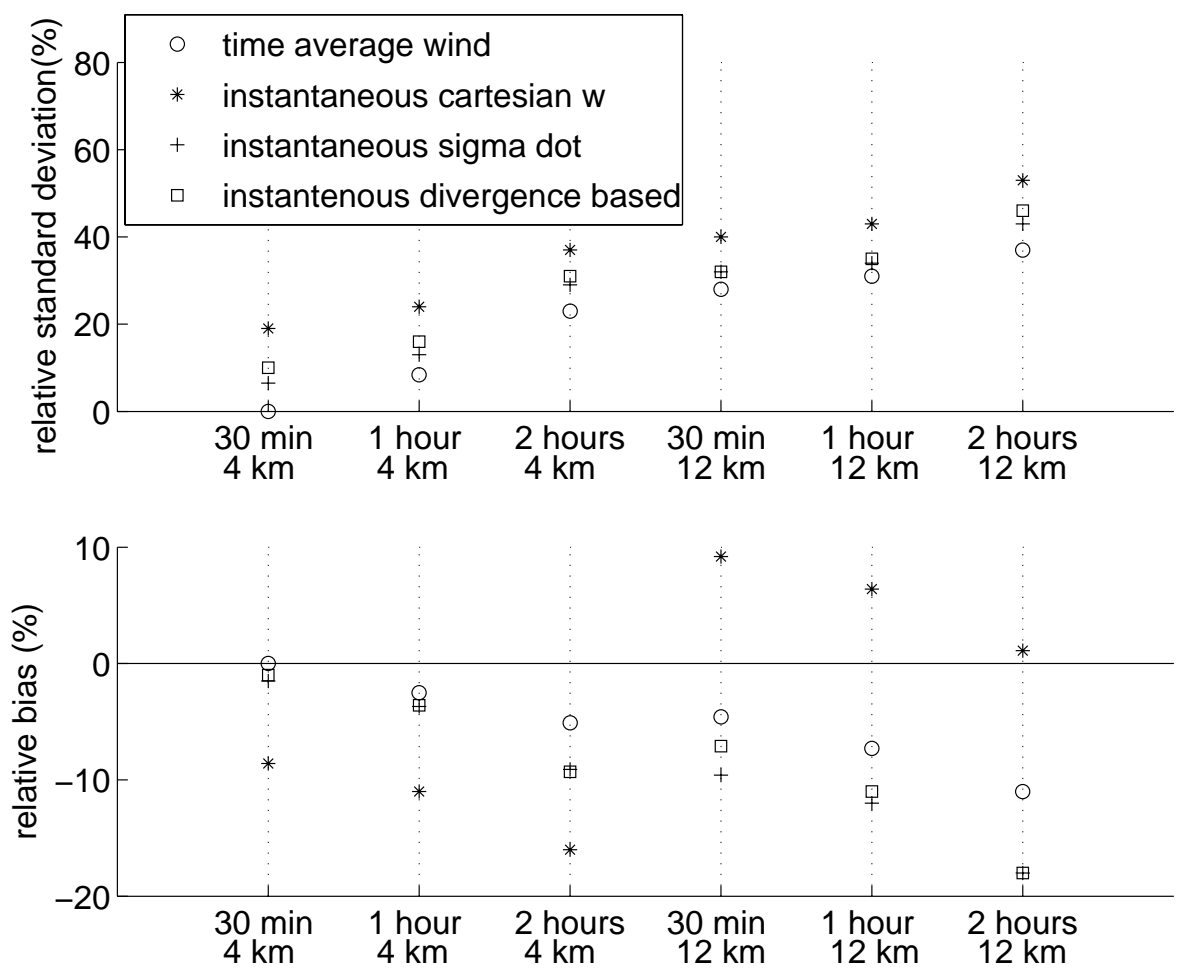

Fig. 3. Relative standard deviation (top) and relative bias (bottom) for different experiments using footprints from the run at 30 min time interval output and $4 \mathrm{~km}$ horizontal resolution that uses time-average wind as reference. See Table 2 and Sect. 3.3 for details.

The most significant differences are found when using the $2 \mathrm{~h}$ time interval output. The bias using instantaneous wind is about 2 times larger than using time average wind.

The values of relative bias and uncertainty using instantaneous wind with $W_{f}$ are equivalent to those using $\dot{\sigma}$.

The largest uncertainties and biases are found using the geometric cartesian vertical velocity $w$. Uncertainty varies from $19 \%-53 \%$, and relative bias varies from $-16 \%$ to $+9.2 \%$. The relative bias becomes systematically positive when using $12 \mathrm{~km}$ resolution output. We did not investigate in detail the reason. For a typical output interval of $1 \mathrm{~h}$, the relative standard deviation is $24 \%$ at $4 \mathrm{~km}$ resolution and $43 \%$ at $12 \mathrm{~km}$ resolution, while the relative bias is $-11 \%$ and $+6.4 \%$, respectively.

Figure 4 presents maps of relative biases at $1 \mathrm{~h}$ time interval output and $4 \mathrm{~km}$ resolution. Those plots are representative of the geographical distribution of biases using other time interval outputs and resolutions. As expected, the largest bias in each grid cell is found using instantaneous wind with $w$, and the smallest using time average winds. A gradient of bias is visible, directed from the ocean toward the continent for each run. It is not related to any boundary effect in the FLEXPART domain, but due to differences in orography. Therefore, results found in Fig. 3 or Table 2 are valid for complex terrain, but are not necessarily applicable to domains with flat terrain.

Figure 5 presents the relationship between relative biases and average gradient of orography calculated within $16 \mathrm{~km}$ of each location with a time interval output of $1 \mathrm{~h}$ and $4 \mathrm{~km}$ resolution. Figure 6 presents the relationship between relative standard deviation and average gradient of orography. Linear relationships are correlated enough to fit a linear slope across.

For an average orographic gradient of $40 \mathrm{~m} \mathrm{~km}^{-1}$, typically found near mountains, the bias is $-5.7 \%$ for time average wind, $-6.6 \%$ for instantaneous wind with $\dot{\sigma},-8.7 \%$ for instantaneous wind with $W_{f}$ and $-28 \%$ for instantaneous wind with $w$ (relative standard deviation of $7.5 \%, 10 \%$, $15 \%$ and $30 \%$, respectively). In flat terrain situation (orography gradient close to $0 \mathrm{~m} \mathrm{~km}^{-1}$ ), the biases only vary from -0.5 to $-1.4 \%$ among the 4 runs (relative standard deviation varying from $5 \%$ to $10 \%$ ). Numerical uncertainties increase with orography because the gradient of orography becomes more uncertain and subgrid wind variability increases. It is also the reason why uncertainties in Table 2 increase with the resolution and time interval output.

As expected, uncertainties found in complex terrain become much smaller in flat terrain, and differences in uncertainty and bias using time average wind or instantaneous wind with $w$ or $\dot{\sigma}$ become much smaller. It demonstrates that instantaneous wind with $w$ can be used in Lagrangian models as long as the local gradient of orography stays small. Instantaneous wind with $\dot{\sigma}$ can be used in complex terrain with small uncertainty involved as long as the time interval output is small (typically $1 \mathrm{~h}$ or less). 
A)

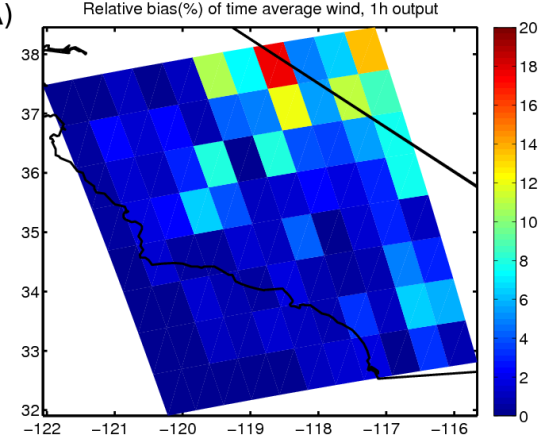

C) Relative bias(\%) of instantaneous wind, eta dot, 1h output

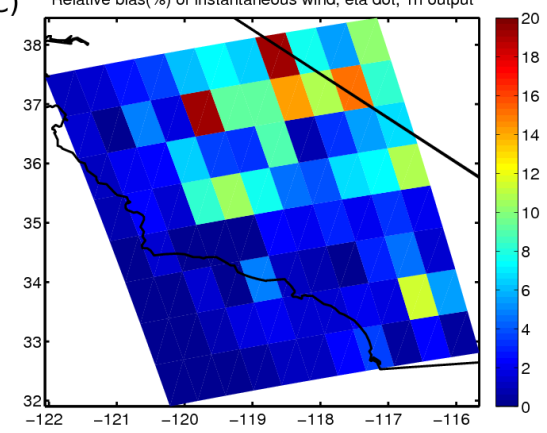

B)

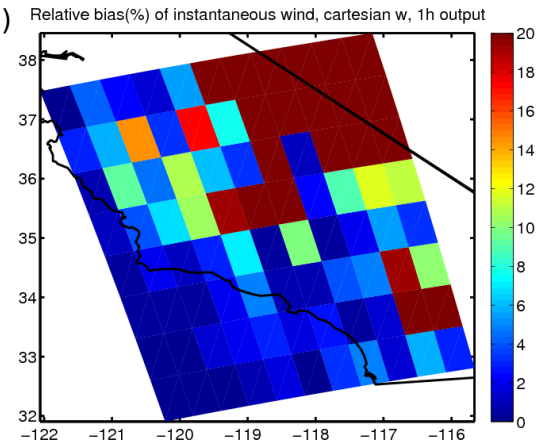

D) Relative bias $\%$ of instantaneous wind, divergence based $w$, th output

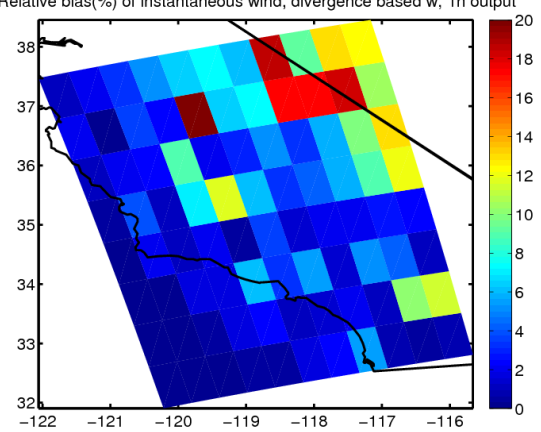

Fig. 4. Maps of relative bias (\%) using (a) time average wind, (b) instantaneous wind with cartesian vertical velocity, (c) instantaneous wind with sigma dot, and (d) instantaneous wind with divergence based vertical velocity with $1 \mathrm{~h}$ time interval output and $4 \times 4 \mathrm{~km}$ resolution.

\section{Discussion and conclusion}

We have shown differences in wind divergences when using the geometric cartesian vertical velocity with instantaneous wind $(w)$ compared to using sigma $\operatorname{dot}(\dot{\sigma})$ or time average $\dot{\sigma}$ out of the WRF mesoscale model. No simple method can be used to correctly convert the geometric cartesian vertical velocity onto terrain following coordinates in complex terrain. As a result, wind is not mass balanced when using $w$. As shown in Sect. 3.3, the mass within the model using instantaneous wind with $w$ can vary by $25 \%$ within $24 \mathrm{~h}$, and the residence time within grid cells can be uncertain by 19$53 \%$, depending on the horizontal resolution or time interval output. However, in flat terrain, those uncertainties become much smaller and therefore the geometric cartesian vertical velocity with instantaneous wind can be used in Lagrangian models as long as the variation in orography within the domain of interest stays small. Uncertainties in the inversion technique used in Brioude et al. $(2011,2012)$ in the Houston region by using instantaneous wind with geometric cartesian vertical velocity can be evaluated to about $10 \%$, smaller than typical uncertainties in PBL mixing height (about 20\%). Using a wind divergence based vertical velocity $\left(W_{f}\right)$, the mass conservation and uncertainty improve compared to $w$, but is less accurate than $\dot{\sigma} . W_{f}$ can be used as an alternative to $w$ if no other options are available.

Mass conservation in the FLEXPART model was not an issue when using instantaneous wind with $\dot{\sigma}$. The use of time- average wind did not significantly improve it. However, uncertainty and bias in the trajectory calculations are systematically better when using time-average wind compared to instantaneous wind, but not as crucial as what was thought before. For a typical output interval of $1 \mathrm{~h}$, the uncertainty improves from $13 \%-8.4 \%$ at $4 \mathrm{~km}$ resolution $(34 \%-31 \%$ at $12 \mathrm{~km}$ resolution) and the bias improves from $-3.7 \%$ to $-2.5 \%$ at $4 \mathrm{~km}$ resolution $(-12 \%$ to $-7.3 \%$ at $12 \mathrm{~km}$ resolution) in complex terrain. Differences in uncertainty and bias are even smaller in flat terrain.

Time-average wind reduces significantly the bias (by a factor of 2) when using a $2 \mathrm{~h}$ time interval output. However, the reduction in relative uncertainty is much smaller. This is probably because the time variability in the vertical wind is much bigger than in the horizontal wind, which means that the error in instantaneous vertical wind is proportionally bigger than in the horizontal wind. Therefore, time average wind is necessary to capture adequately the time variability in the vertical velocity for a time interval output of $2 \mathrm{~h}$ or larger. However, at $1 \mathrm{~h}$ time interval output or less, this difference is fairly small and should not prevent the use of instantaneous wind in Lagrangian models, even though the use of time-average wind would always be beneficial in reducing the uncertainties in the model. Of course, the relationships between bias or uncertainty with time interval output will depend on the horizontal resolution. For the frontal case of Grell et al. (2004), about $50 \%$ of the variability in the vertical 

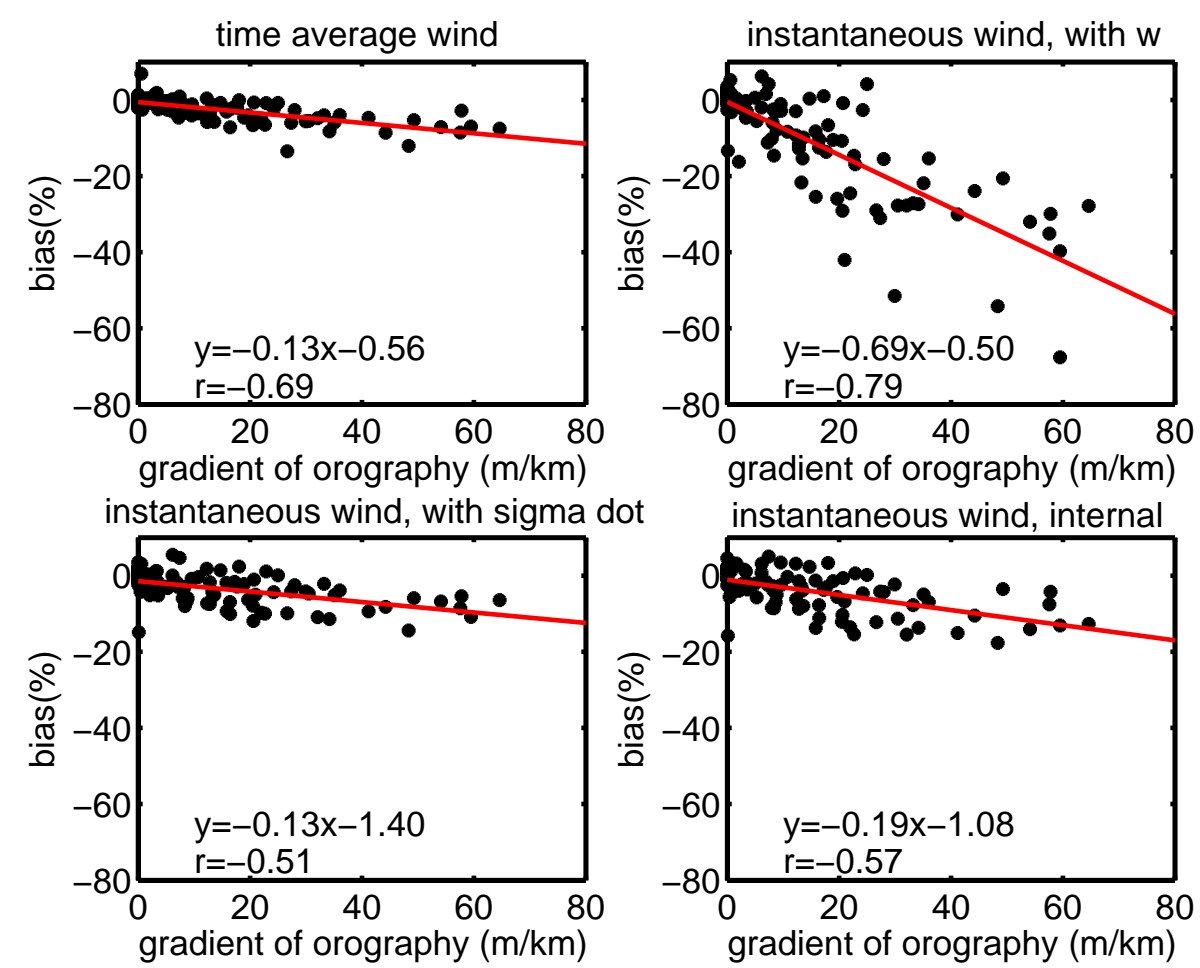

Fig. 5. Linear relationship between relative bias $(\%)$ and average gradient of orography at a particular grid cell for experiments with $1 \mathrm{~h}$ output and $4 \mathrm{~km}$ horizontal resolution using (a) time average wind, (b) instantaneous wind with $w$, (c) instantaneous wind with $\dot{\sigma}$, and (d) instantaneous wind with divergence based vertical velocity $\left(W_{f}\right)$. See the text for details.
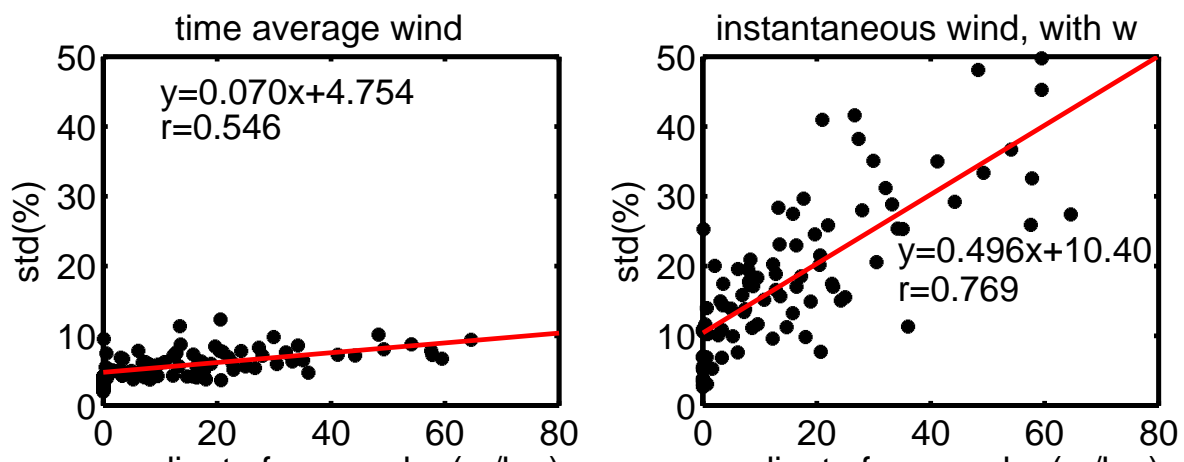

gradient of orography $(\mathrm{m} / \mathrm{km})$

gradient of orography $(\mathrm{m} / \mathrm{km})$
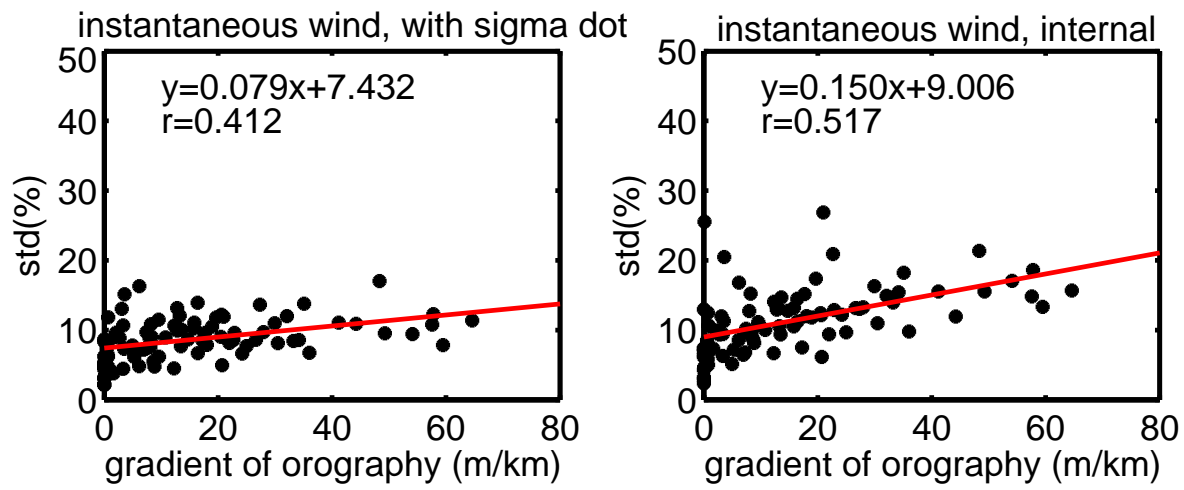

Fig. 6. Same Fig. 5, but valid for relative standard deviation. 
wind can be captured with a time interval output of $30 \mathrm{~min}$, and $30 \%$ at $1 \mathrm{~h}$ at 3 or $9 \mathrm{~km}$ resolution.

For a typical time interval output of $1 \mathrm{~h}$ and $4 \mathrm{~km}$ resolution, the use of time average winds reduced the relative bias by $1.2 \%$ and relative uncertainty by $5 \%$ compared to instantaneous wind with $\dot{\sigma}$ in complex terrain. While it is always good to reduce numerical uncertainties, we found that using time-average wind is not as crucial as has been thought. When using a Lagrangian model for an application like chemical forecast or top-down estimate of emission inventories, a larger source of uncertainty is the PBL height, which is typically about $20 \%$. The quality of Lagrangian trajectories will probably be more affected by the quality of the wind simulation from the Eulerian model than the benefit of using time-average wind compared to instantaneous wind.

Acknowledgements. The ERA-interim data for this study are from the Research Data Archive, which is maintained by the Computational and Information Systems Laboratory at the National Center for Atmospheric Research. The original data are available from the RDA (http://dss.ucar.edu) in data set number ds627.0.

Edited by: V. Grewe

\section{References}

Angevine, W. M., Eddington, L., Durkee, K., Fairall, C., Bianco, L., and Brioude, J.: Meteorological model evaluation for CalNex 2010, Mon. Wea. Rev., doi:10.1175/MWR-D-12-00042.1, in press, 2012.

Brioude, J., Kim, S. -W., Angevine, W. M., Frost, G. J., Lee, S. H., McKeen, S. A., Trainer, M., Fehsenfeld, F. C., Holloway, J. S., Ryerson, T. B., Williams, E. J., Petron, G., and Fast, J. D.: Top-down estimate of anthropogenic emission inventories and their interannual variability in Houston using a mesoscale inverse modeling technique, J. Geophys. Res., 116, D20305, doi:10.1029/2011JD016215, 2011.
Brioude, J., Petron, G., Frost, G. J., Ahmadov, R., Angevine, W. M., Hsie, E. -Y., Kim, S. -W., Lee, S. H., McKeen, S. A., Trainer, M., Fehsenfeld, F. C., Holloway, J. S., Peischl, J., Ryerson, T. B., and Gurney, K. R.: A new inversion method to calculate emission inventories without a prior at mesoscale: application to the anthropogenic $\mathrm{CO}_{2}$ emission from Houston, Texas, J. Geophys. Res., 117, D05312, doi:10.1029/2011JD016918, 2012.

Fast, J. D. and Easter, R.: A Lagrangian Particle Dispersion Model Compatible with WRF, in: 7th WRF User's Workshop, National Center for Atmospheric Research, June 19-22, Boulder, CO, 6.2, 2006.

Grell, G. A., Knoche, R., Peckham, S. E., and McKeen, S. A.: Online versus offline air quality modeling on cloud-resolving scales, Geophys. Res. Lett., 31, L16117, doi:10.1029/2004GL020175, 2004.

Hanna, S. R.: Applications in air pollution modeling, in: Atmospheric Turbulence and Air Pollution Modelling, edited by: Nieuwstadt, F. T. M. and van Dop, H., D. Reidel Publishing Company, Dordrecht, 1982.

Lin, J. C., Gerbig, C., Wofsy, S. C., Daube, B. C., Andrews, A. E., Davis, K. J., and Grainger, C. A.: A near-field tool for simulating the upstream influence of atmospheric observations: the stochastic time-inverted lagrangian transport (STILT) model, J. Geophys. Res., 108(D16), 4493, doi:10.1029/2002JD003161, 2003.

Nehrkorn, T., Eluszkiewicz, J., Wofsy, S. C., Lin, J. C., Gerbig, C., Longo, M. and Freitas, S.: Coupled weather research and forecasting - stochastic time-inverted lagrangian transport (WRF-STILT) model, Meteorol. Atmos. Phys., 107, 51-64, doi:10.1007/s00703-010-0068-x, 2010.

Simmons, A., Uppala, S., Dee, D., and Kobayashi, S.: ERA-Interim: New ECMWF reanalysis products from 1989 onwards, Newsl. 110, 11 pp., Eur. Cent. for Medium-Range Weather Forecasts, Reading, 2007.

Skamarock, W. C., Klemp, J. B., Dudhia, J., Gill, D. O., Barker, D. M., Duda, M. G., Hwang, X.-Y., Wang, W., and Powers, J. G.: A description of the advanced research WRF version 3, Technical Note 475+STR, National Center for Atmospheric Research, Boulder, CO, USA, 2008.

Stohl, A.: Computation, accuracy and applications of trajectories a review and bibliography, Atmos. Environ. 32, 947-966, 1998.

Stohl, A., Forster, C., Frank, A., Seibert, P., and Wotawa, G.: Technical note: The Lagrangian particle dispersion model FLEXPART version 6.2, Atmos. Chem. Phys., 5, 2461-2474, doi:10.5194/acp-5-2461-2005, 2005. 